\title{
Gráficos e equações: a articulação de dois registros ${ }^{1}$
}

\author{
Graphs and equations: articulating two registers
}

\author{
Raymond Duval \\ Professor emérito da Université du Littoral Côte d'Opale/France
}

Tradução: Méricles Thadeu Moretti

PPGECT/MTM/UFSC

mericles@mtm.ufsc.br

\begin{abstract}
Resumo
A leitura das representações gráficas requer dos alunos a discriminação das diferentes variáveis visuais pertinentes constituintes deste tipo de representação. Requer também que os alunos tenham consciência das correspondências entre as variações visuais dos gráficos e as alterações significativas na escrita algébrica da relação. Este modo de pensar a leitura das representações gráficas vai na contramão da prática corrente, a qual utiliza regras de codificação para construir representações gráficas fundamentadas na associação entre pares ordenados de números e pontos do plano. Isto leva a um abismo cognitivo entre a interpretação global, que se espera dos alunos, e a leitura ponto a ponto na qual o professor se baseia para introduzir o registro gráfico. Neste trabalho, descreveremos as variáveis visuais que são matematicamente pertinentes para a interpretação global das representações gráficas e, conforme o princípio experimental que isola cada variável visual para observar as variações correspondentes no segundo registro, construímos um conjunto de atividades de reconhecimento. Os resultados obtidos, com diferentes grupos de aluno, mostram que a maioria deles, mesmo após um ensino da noção de função em nível de seconde $e^{2}$, não distingue as variáveis visuais significativas nos gráficos, visualmente muito diferentes umas das outras, e nem consegue articular estas variáveis com as expressões algébricas correspondentes.
\end{abstract}

\section{Résumé}

La lecture des représentations graphiques requiert des étudiant qu'ils discriminent les différentes variables visuelles pertinentes constituant ce type de représentation et qu'ils prennent conscience des correspondances entre les variations visuelles des graphes et les changements significatifs dans l'écriture algébrique de la relation. Cela va contre la pratique courante qui est induite par la règle de codage utilisée pour construire les représentations graphiques, laquelle se fonde sur l'association un couple de nombre pour un point du plan quadrillé. Il en résulte un fossé cognitif entre l'interprétation globale qui est attendue des élèves et la lecture ponctuelle à laquelle l'enseignement s'en tient pour introduire le registre des représentations graphiques. Dans ce papier nous allons décrire toutes variables visuelles qui sont mathématiquement pertinentes pour une interprétation globale des représentations graphiques. Et, selon le principe expérimental, en isolant chaque variable visuelle pertinente pour observer les co-variations correspondantes dans le second registre, nous avons construit un ensemble de tâches de reconnaissance. Les résultats obtenus auprès de différentes populations

\footnotetext{
${ }^{1}$ Este texto é uma tradução do artigo: DUVAL, R. Graphiques et équations: L'articulation de deux registres. Annalles de Didactiques et de Sciences Cognitives. v.1. Strasbourg: ULP - IREM, 1988. Algumas adaptações de forma no texto original foram produzidas para adequar as normas da ABNT além do resumo do texto reformulado pelo autor.

${ }^{2}$ N. do Tradutor. No ensino básico francês, seconde corresponde ao $10^{\circ}$ ano de escolaridade. Depois da série de seconde, tem-se as séries premier e terminale. Essas três séries correspondem aproximadamente as três séries do ensino médio no Brasil. Neste texto será mantido a mesma denominação das séries em francês.
} 
montrent que la grande majorité des élèves, après un enseignement de la notion de fonction au niveau de la seconde $^{3}$, ne distinguent pas les différentes variables visuelles significatives dans des graphes visuellement très différents et ne les articulent pas avec les expressions algébriques correspondantes.

\begin{abstract}
The reading of graphical representations requires students to discriminate all the relevant visual variables, which make this kind of representation and to realize the correspondences between qualitative variations of graphs and significant changes in the algebraic expression. That goes against the current practice induced by coding construction of graphs and graphics, which is, based on the association a point with a pair of numbers. The outcome is a cognitive gap between the global mathematical interpretation expected from students and the local reading highlighted in teaching for introducing graphical representations. In this paper we describe all the visual variables, which are mathematically relevant for the global interpretation of graphical representations. And applying the experimental principal of isolating each relevant visual variable for observing the corresponding covarations in the other register, we have constructed a set of recognition tasks. The results obtained from different populations show that most students, after a teaching about function at the level of seconde ${ }^{4}$, neither distinguish these visual variables in graphs, yet visually quite different, nor articulate them with algebraic expressions.
\end{abstract}

\title{
Introdução
}

Muitos estudos apontam dificuldades de leitura e de interpretação das representações gráficas cartesianas. Por exemplo, a ligação entre o conceito de inclinação ${ }^{5}$ e direção da reta no plano não é em geral efetuada. (HERSCOVICS, 1980). Também a confusão entre inclinação e altura parece ser frequente. (CLEMENT, 1985). Observa-se ainda a impossibilidade de encontrar a equação de uma reta partindo de sua representação gráfica, até para os casos mais elementares. Mesmo para o caso das retas, a articulação entre o registro das representações gráficas e das equações parece não se estabelecer mesmo depois que os alunos tenham tido aulas sobre funções afins.

A razão profunda dessas dificuldades não se deve procurar nos conceitos matemáticos ligados à função afim, mas na falta de conhecimento das regras de correspondência semiótica entre o registro da representação gráfica e o registro da expressão algébrica. De fato, o ensino e mesmo certos estudos didáticos, atém-se a passagem da equação para a sua representação gráfica com a construção ponto a ponto, esquece-se que é a passagem inversa que traz problema. Para efetuar tal passagem, a abordagem ponto a ponto não é somente inadequada como constitui um obstáculo e, para caracterizá-lo, iremos sucessivamente:

- Apresentar os três tratamentos heterogêneos aos quais as representações cartesianas provocam;

\footnotetext{
${ }^{3}$ Ver nota 2 de rodapé.

${ }^{4}$ Ver nota 2 de rodapé.

${ }^{5}$ No caso deste estudo, nos limitaremos aos sistemas ortogonais. Para os sistemas não ortogonais seria necessário considerar não mais a noção de inclinação, mas de coeficiente diretor.
} 
- Explicitar as variáveis visuais pertinentes que correspondem às características significativas na expressão algébrica;

- Ilustrar, a nossa análise, com alguns resultados de uma enquete levada a cabo para este fim e, também, para explicitar erros típicos identificados em outros estudos.

Pelo fato de não se considerar as regras semióticas de correspondência, as representações gráficas tornam-se representações obscuras para a maioria dos alunos do seconde e para além desse nível.

\section{Os três tratamentos das representações gráficas}

Três abordagens são possíveis para a representação gráfica: elas não levam em conta os mesmos dados visuais do gráfico e não são guiadas pelo mesmo tipo de questão.

\subsection{A abordagem ponto a ponto}

É por meio desta abordagem que são introduzidas e definidas as representações gráficas. Em referência aos dois eixos graduados, um par de números permite identificar um ponto (e, inversamente, um ponto se traduz por um par de números). Este modo associativo limita-se a alguns valores particulares e aos pontos marcados no plano referencial. Esta abordagem favorece quando se quer TRAÇAR o gráfico correspondente de uma equação do primeiro grau ou o gráfico de uma equação do segundo grau. Favorece ainda quando se quer LER as coordenadas de algum ponto interessante (porque é ponto de intersecção com os eixos ou com alguma reta, porque é máximo, etc.).

\subsection{Uma abordagem de extensão do traçado efetuado}

Esta abordagem corresponde às atividades de interpolação e extrapolação aos quais se apóiam o que se denominou de aspectos produtores ou redutores das representações gráficas. (ROGALSKI, 1981). De modo geral, esta abordagem de extensão se mantém puramente mental: ela não acarreta traços complementares e explicativos como uma mudança local na graduação dos eixos para ampliar uma parte do traçado. É preciso assinalar que esta abordagem de extensão não se atém mais sobre um conjunto finito de pontos marcados (a intersecção das linhas em papel milimetrado, por exemplo) como no caso da abordagem ponto a ponto; esta extensão se apóia em um conjunto infinito de pontos potenciais, quer dizer, no fundo homogêneo da folha, nos intervalos entre pontos marcados. Mas nesta abordagem, 
como no caso da precedente, levam-se em conta os dados do traçado e não as variáveis visuais pertinentes da representação gráfica. No mais, o tratamento se mantém orientado na busca de valores particulares sem se ocupar com a forma da expressão algébrica.

\subsection{Abordagem de interpretação global de propriedades figurais}

O conjunto traçado/eixos forma uma imagem que representa um objeto descrito por uma expressão algébrica. Toda modificação desta imagem, que leva a uma modificação na expressão algébrica correspondente, determina uma variável visual pertinente para a interpretação gráfica. É importante, deste modo, identificar todas as modificações pertinentes possíveis desta imagem, quer dizer, ver as modificações conjuntas da imagem e da expressão algébrica: isto significa proceder a uma análise de congruência entre dois registros de apresentação de um objeto ou de uma informação. Com esta abordagem não estamos mais na presença da associação "um ponto - um par de números", mas na presença da associação "variável visual de representação - unidade significativa da expressão algébrica".

Quando se trata de partir da representação gráfica para encontrar, por exemplo, a equação correspondente ou para utilizar o conceito de inclinação ou de direção, é esta abordagem de interpretação global que se torna necessária. A razão disto se deve ao fato de que o recurso à abordagem ponto a ponto é totalmente inoperante uma vez que tira a atenção das variáveis visuais. A prática sistemática da abordagem ponto a ponto não favorece a abordagem de interpretação global que é em geral deixada de lado no ensino uma vez que depende de análise semiótica visual e algébrica. Compreende-se porque a maioria dos alunos fica aquém de uma utilização correta das representações gráficas.

\section{Variáveis visuais e unidades simbólicas significativas}

Uma análise de congruência exige a discriminação das unidades significativas próprias a cada registro de apresentação assim como o exame das transformações implícitas eventuais exigidas para mudar de registro.

A discriminação das unidades significativas próprias a uma expressão algébrica é relativamente evidente. Tem-se: 
- Os símbolos relacionais $(<,>,=, \ldots)$;

- Os símbolos de operações ou de sinais $(+,-)$;

- Os símbolos de variável;

- Os símbolos de expoente, coeficiente e de constante.

Em uma expressão algébrica, cada símbolo corresponde geralmente a uma unidade significativa. Existe, no entanto, unidades significativas em que os símbolos são omitidos: o coeficiente 1, o caráter positivo dos coeficientes maiores do que zero. Deste modo, não se escreve $\mathrm{y}=+1 \mathrm{x}$, em contrapartida, escreve-se $\mathrm{y}=-2 \mathrm{x}$. O lembrete desta trivialidade é importante uma vez que se trata de fazer corresponder variáveis visuais pertinentes do gráfico com unidades significativas da expressão algébrica.

A descriminação das propriedades figurais de uma representação gráfica é, em contrapartida, menos evidente. Tomando certas variáveis visuais definidas por Bertin (1977) e especificando outras, distinguiremos duas variáveis gerais e três variáveis relativas para o caso em que o gráfico é um traçado simples (reta ou parábola).

As duas variáveis gerais são relacionadas ao:

- De implantação da tarefa, quer dizer, o que se destaca como figura sobre o fundo: uma linha ou uma zona;

- Relativa à forma da tarefa: a linha traçada que delimita ou não uma zona é reta ou curva. Se for curva, ela é aberta ou fechada.

As três variáveis particulares correspondem a uma simples modificação de configuração linha traçada/eixos orientados. Limitamo-nos aqui ao caso em que a linha é uma reta. Uma análise bastante similar pode ser efetuada para o caso em que a linha é uma curva aberta como no caso de uma parábola. Na tabela a seguir, acomodamos essas três variáveis visuais. 
Tabela 1: Valores e variáveis visuais para a reta no plano cartesiano.

\begin{tabular}{|l|l|}
\hline \multicolumn{1}{|c|}{ Variáveis visuais } & \multicolumn{1}{c|}{ Valores das variáveis visuais } \\
\hline - o sentido da inclinação do traçado: & $\begin{array}{l}\text { - a linha sobe da esquerda para a direita; } \\
\text { - a linha desce da esquerda para a direita. }\end{array}$ \\
& $\begin{array}{l}\text { OBSERVAÇão: a referência esquerda/direita é o sentido normal do } \\
\text { percurso visual de uma página escrita em caracteres latinos. }\end{array}$ \\
\hline - os ângulos do traçado com os eixos: & $\begin{array}{l}\text { Há uma repartição simétrica do quadrante } \\
\text { percorrido. } \\
\text { - o ângulo formado com o eixo horizontal é menor } \\
\text { que o ângulo formado com o eixo vertical; }\end{array}$ \\
& $\begin{array}{l}\text { - o ângulo formado com o eixo horizontal é maior } \\
\text { que o ângulo formado com o eixo vertical; }\end{array}$ \\
& $\begin{array}{l}\text { OBSERvAção: no caso em que o traçado não passa pela origem, } \\
\text { basta deslocar o eixo vertical, por exemplo, até o ponto de } \\
\text { intersecção da reta com o eixo horizontal. }\end{array}$ \\
\hline - a posição do traçado em relação à \\
origem do eixo vertical:
\end{tabular}

A primeira das três variáveis visuais particulares pode tomar dois valores, a segunda, três valores, e a terceira também três valores. Omitimos dois casos em que a reta é paralela a um dos eixos: não é necessário levar em conta os valores das variáveis precedentes, basta ler o valor do ponto de intersecção da reta com o eixo.

A cada uma dessas oito variáveis visuais particulares corresponde uma unidade significativa na expressão algébrica da reta: o que importa na expressão $y=a x+b$ é o coeficiente a e a constante b (Tabela 2 a seguir).

Tabela 2: Valores e variáveis visuais para $\mathrm{y}=\mathrm{ax}+\mathrm{b}$ no plano cartesiano.

\begin{tabular}{|l|l|l|l|}
\hline \multicolumn{1}{|c|}{ Variáveis visuais } & \multicolumn{1}{c|}{ Valores } & \multicolumn{2}{c|}{ Unidades simbólicas correspondentes } \\
\hline Sentido da inclinação & $\begin{array}{l}\text { ascendente } \\
\text { descendente }\end{array}$ & $\begin{array}{l}\text { coeficiente }>0 \\
\text { coeficiente <0 }\end{array}$ & $\begin{array}{l}\text { ausência de sinal } \\
\text { presença do sinal - }\end{array}$ \\
\hline Ângulo com os eixos & $\begin{array}{l}\text { partição simétrica } \\
\text { ângulo menor } \\
\text { ângulo maior }\end{array}$ & $\begin{array}{l}\text { coefic. variável =1 } \\
\text { coefic. variável <1 } \\
\text { coefic. variável }>1\end{array}$ & $\begin{array}{l}\text { não há coefic. escrito } \\
\text { há coefic. escrito } \\
\text { há coefic. escrito }\end{array}$ \\
\hline Posição sobre o eixo & $\begin{array}{l}\text { corta acima } \\
\text { corta abaixo } \\
\text { corta na origem }\end{array}$ & $\begin{array}{l}\text { acresc. constante } \\
\text { subtrai-se constante } \\
\text { sem correção aditiva }\end{array}$ & $\begin{array}{l}\text { sinal + } \\
\text { sinal - } \\
\text { ausência de sinal }\end{array}$ \\
\hline
\end{tabular}

A simples leitura deste quadro, limitada ao caso das retas, permite que façamos as seguintes observações:

- O conceito de inclinação, algebricamente traduzido pelo coeficiente, recobre duas unidades significativas diferentes: uma definida em relação ao sinal e a outra em 
relação ao inteiro 1. Estas duas unidades significativas correspondem a duas variáveis diferentes, respectivamente, o sentido da inclinação e ao ângulo. Não há congruência entre a direção da reta no plano cartesiano e o coeficiente que determina esta direção na expressão algébrica, uma vez que para qualquer valor do coeficiente dado $(2,1 / 2,-2$, etc.) é necessário destacar duas propriedades distintas relativamente ao 0 e ao 1 .

- O custo muito desigual na passagem entre a expressão na forma simbólica e a representação gráfica aparece aqui de modo preciso. Para passar da escrita simbólica para a representação gráfica, é possível se contentar com a abordagem ponto a ponto: atribuem-se valores particulares a x sem se preocupar com quaisquer propriedades para encontrar pares de números, quer dizer, pontos. Mas, para passar da representação gráfica para a expressão algébrica, isto não é possível: é preciso identificar cada um dos valores das variáveis visuais e integrá-las. Dito de outro modo, a passagem da representação gráfica para a expressão algébrica exige uma interpretação global. Diferentemente da abordagem ponto a ponto, ou mesmo da abordagem de extensão representativa, a abordagem de interpretação global exige que a atenção esteja centrada sobre um conjunto de propriedades e não sobre valores particulares tomados um a um.

- Vê-se que, para as retas não paralelas aos eixos, há somente 18 representações gráficas que são visualmente diferentes de modo significativo. A cada uma dessas representações corresponde uma equação particular (ver Tabela 3 a seguir):

Tabela 3: Identificação e integração, com exemplos, de 18 representações de variáveis visuais.

\begin{tabular}{|c|c|c|c|}
\hline \multirow[t]{4}{*}{ Sentido da inclinação } & ângulo & Posição (da reta) & Exemplos \\
\hline & & $\begin{array}{l}\text { (na origem) } \\
+ \text { (acima da origem }) \\
-(\text { abaixo da origem })\end{array}$ & $\begin{array}{l}y=x \\
y=x+1 \\
y=x-1\end{array}$ \\
\hline & $>1$ & $\begin{aligned} & (\text { na origem }) \\
+ & (\text { acima da origem }) \\
- & (\text { abaixo da origem })\end{aligned}$ & $\begin{array}{l}y=2 x \\
y=2 x+1 \\
y=2 x-1\end{array}$ \\
\hline & $<1$ & $\begin{aligned} & (\text { na origem }) \\
+ & (\text { acima da origem }) \\
- & \text { (abaixo da origem })\end{aligned}$ & $\begin{array}{l}y=(1 / 2) x \\
y=(1 / 2) x+1 \\
y=(1 / 2) x-1\end{array}$ \\
\hline$<0$ & & & \\
\hline
\end{tabular}


No caso de paralelismo a um dos eixos, desaparece a variável a que se refere tal eixo.

Uma apresentação explícita e sistemática das variações visuais significativas não somente centra a atenção sobre a correspondência entre a representação gráfica e expressão algébrica, mas permite encontrar diretamente a expressão algébrica das propriedades geométricas: perpendicularidade e paralelelismo de duas retas, por exemplo. De fato, é suficiente praticar a abordagem experimental a mais clássica: variar uma unidade significativa na expressão, mantendo as outras constantes e ver o que se passa no outro registro (ou mudar uma variável visual mantendo as duas outras constantes e ver as modificações que acontecem na expressão). Assim, por exemplo, a oposição entre $y=x$ e $y=-x$ se articula em uma unidade de uma imagem visual e esta imagem se presta a modificações que têm contrapartida algébrica imediata.

Esta análise não se limita ao caso das retas. Retornando às duas variáveis gerais mencionadas anteriormente, podemos indicar as correspondências seguintes:

Tabela 4: Características visuais de representação no plano cartesiano.

\begin{tabular}{|c|c|c|}
\hline Variáveis visuais & Valores & Unidades simbólicas correspondentes \\
\hline $\begin{array}{l}\text { - implantação da tarefa } \\
\text { (o que se destaca como } \\
\text { figura sobre o fundo). }\end{array}$ & $\begin{array}{l}\text { - zona } \\
\text { - linha }\end{array}$ & $\begin{array}{c}>,<, \ldots \\
=\end{array}$ \\
\hline $\begin{array}{l}\text { - forma da tarefa } \\
\text { (a linha traçada delimita ou } \\
\text { não uma zona aberta ou } \\
\text { fechada). }\end{array}$ & $\begin{array}{l}\text { - linha reta } \\
\text { - linha curva }\end{array}$ & $\begin{array}{l}\text { expoente da variável }=1 \\
\text { expoente da variável }>1\end{array}$ \\
\hline
\end{tabular}

Levando em conta de forma implícita ou explícita a expressão das funções, poderemos integrar outras características visuais importantes, como o caráter aberto ou fechado das curvas $^{6}$. Sem desenvolver aqui esta análise, podemos simplesmente relevar que estas variáveis gerais, diferentemente das variáveis particulares, correspondem às modificações intrínsecas da imagem.

Apesar das aparências, tal abordagem de articulação entre representação gráfica e expressão algébrica não está presente nas perspectivas de ensino de matemática. $\mathrm{O}$ exame, por exemplo, de um manual didático recente nos surpreende pela variedade e riqueza de representações gráficas, apresenta um "herbário de funções usuais" (Mathématiques $2^{\text {ème }}$, p. 323) que nos dá a impressão de que há uma preocupação com a articulação entre os registros gráfico e

${ }^{6}$ Estas possibilidades de extensão de análise de congruência foram sugeridas por F. Pluvinage. 
algébrico. No entanto, olhando mais de perto, percebe-se que se a posição do traçado e o sentido da inclinação deste traçado são tomados em conta, a variável visual do ângulo do traçado é omitida. Além disso, as duas unidades significativas da inclinação na expressão algébrica não são diferenciadas. Dito de outro modo, a discriminação das variáveis visuais e a sua vinculação com as unidades simbólicas correspondentes são de fato ignoradas. A expressão geral $\mathrm{y}=\mathrm{ax}+\mathrm{b}$ é utilizada como base inicial do trabalho e não como forma de integração cognitiva resultante de uma abordagem de interpretação global já descrita neste texto nos itens 1.3 e 2 .

\section{O sincretismo da percepção das representações gráficas nos alunos}

Não pode haver utilização correta das representações gráficas cartesianas sem a discriminação explícita das variáveis visuais pertinentes e sem uma correspondência sistematicamente estabelecida entre os valores dessas variáveis e as unidades significativas da expressão algébrica. Ignorando a especificidade e a importância da abordagem de interpretação global, o professor não consegue atingir o objetivo de uma utilização correta dos gráficos cartesianos para a maioria dos alunos do primeiro ano do ensino médio (15 a 16 anos). Além disso, as pesquisas didáticas deixam de contar com um meio importante de compreensão dos erros observados.

\subsection{Variáveis visuais particulares e representação das retas}

Para ilustrar a importância e a natureza das dificuldades encontradas pela maioria dos alunos, apresentamos um pequeno teste de reconhecimento proposto a três turmas de seconde do primeiro trimestre de 1987 (veja a figura a seguir). Observamos algumas diferenças em relação a Herscovics (1980), em seu estudo sobre a construção de significações para as equações lineares e, a questão do teste sobre gráficos de Hart (1981). A questão do teste não é significativa uma vez que não exige alguma discriminação entre retas cujos valores possíveis das variáveis visuais são comuns, exceto uma, e também porque não propõe de modo simultâneo uma escolha entre várias expressões algébricas próximas: sem a discriminação de todas as variáveis visuais e das variações na expressão algébrica correspondente, não se pode falar apropriadamente de reconhecimento de uma reta e sua equação. 
Designamos por $\mathrm{x}$ a abscissa e por y a ordenada de um ponto $\mathrm{M}$ do plano referencial. Indicar a qual expressão algébrica $(\mathrm{E} 1, \mathrm{E} 2, \ldots, \mathrm{E} 10)$ cada uma das retas D1, D2, ..., D5 correspondem.
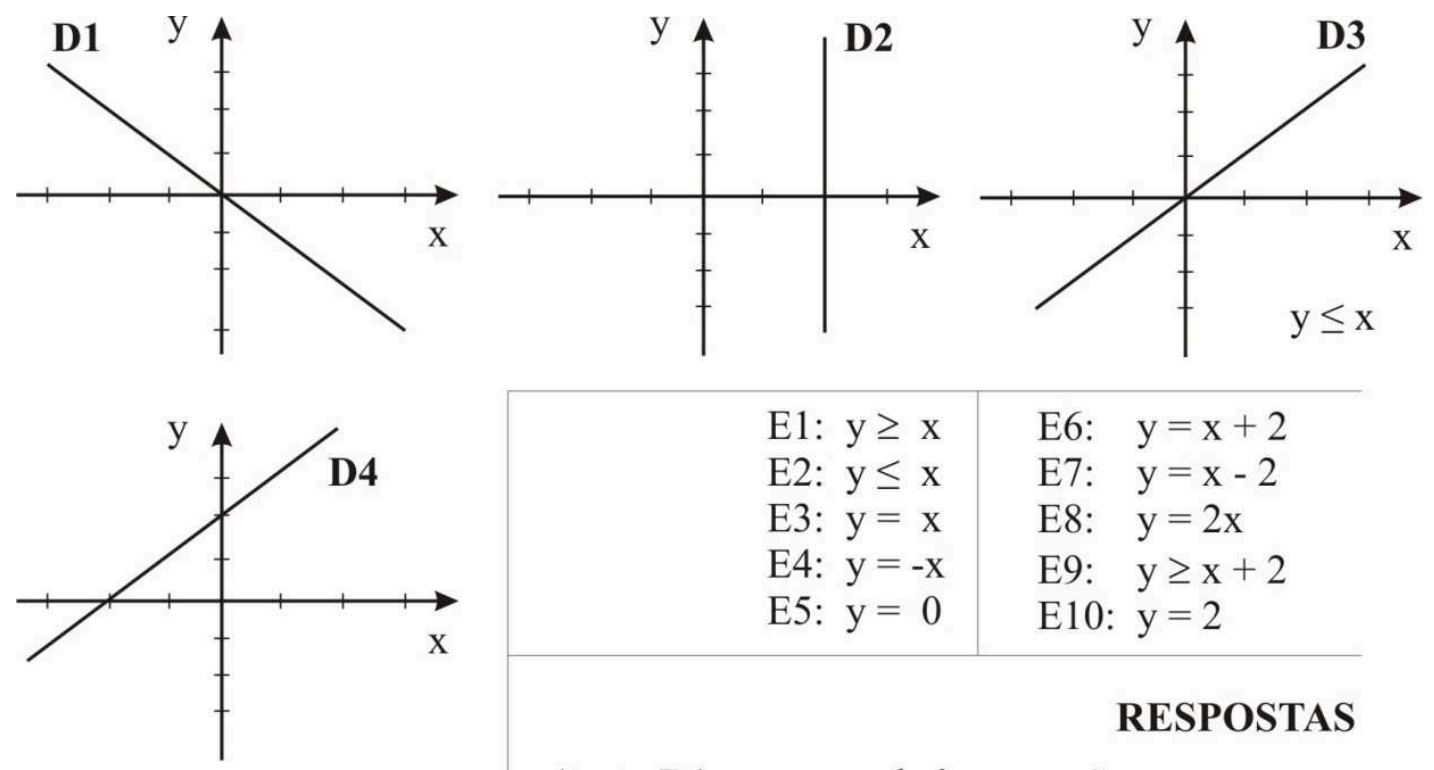

E1: $y \geq x$

E6: $y=x+2$

E2: $y \leq x$

E7: $y=x-2$

E3: $y=x$

E8: $y=2 x$

E4: $y=-x$

E9: $y \geq x+2$

E5: $y=0$

E10: $y=2$

\section{RESPOSTAS}

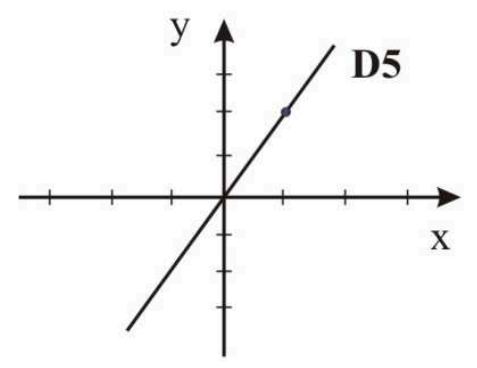

A reta $\mathrm{D} 1$ corresponde à expressão

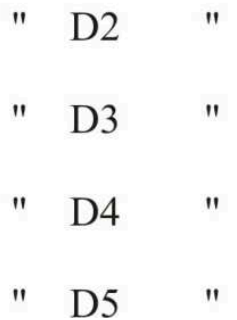

Figura 1: Teste de reconhecimento com retas no plano cartesiano.

De um total de 105 alunos:

$\begin{array}{lll}75 & \text { associaram corretamente } y=x & \text { à } D 3 ; \\ 59 & \text { associaram corretamente } y=-x & \text { à } D 1 ; \\ 68 & \text { associaram corretamente } \mathrm{x}=2 & \text { à } \mathrm{D} 2 ; \\ 26 & \text { associaram corretamente } \mathrm{y}=\mathrm{x}+2 & \text { à } \mathrm{D} 4 ; \\ 39 & \text { associaram corretamente } \mathrm{y}=2 \mathrm{x} & \text { à } \mathrm{D} 5 ; \\ 59 & \text { encontraram e, portanto discriminaram, } \mathrm{y}=\mathrm{x} \text { de } \mathrm{y}=-\mathrm{x} ; \\ 23 & \text { encontraram e, portanto discriminaram, } \mathrm{y}=2 \text { de } \mathrm{y}=\mathrm{x}+2 ; \\ 16 & \text { acertaram os } 5 \text { itens; } \\ 14 & \text { erraram os } 5 \text { itens. }\end{array}$

Entre os erros mais comuns, destacamos: 
- 21 respostas y = x para D1. Estes alunos não associaram a inclinação descendente do traçado ao sinal "-" do coeficiente;

- 23 respostas $y=-x$ para D4. Estes alunos mantiveram apenas a propriedade figural de intersecção de D4 com o lado negativo do eixo x;

- 14 respostas $\mathrm{y} \geq \mathrm{x}$ para D5. Estes 14 alunos procuraram traduzir o fato de que para $\mathrm{o}$ quadrante superior à direita cada valor de y é maior do que o valor de x. Estes alunos manifestamente se prendem a uma abordagem ponto a ponto: diversos pontos são tomados sobre a reta, passa-se aos pares de números associados a estes pontos e procura-se definir uma relação de tal modo que caracterize estas duplas.

Passamos também este teste de reconhecimento em duas outras classes de seconde depois que tiveram um ensino sobre funções afins. Registramos os resultados seguintes para um total de 60 alunos:

$\begin{array}{lll}47 & \text { associaram corretamente } \mathrm{y}=\mathrm{x} & \text { à } \mathrm{D} 3 ; \\ 48 & \text { associaram corretamente } \mathrm{y}=-\mathrm{x} & \text { à } \mathrm{D} 1 ; \\ 49 & \text { associaram corretamente } \mathrm{x}=2 & \text { à } \mathrm{D} 2 ; \\ 24 & \text { associaram corretamente } \mathrm{y}=\mathrm{x}+2 & \text { à } \mathrm{D} 4 ; \\ 27 & \text { associaram corretamente } \mathrm{y}=2 \mathrm{x} & \text { à } \mathrm{D} 5 ; \\ 15 & \text { encontraram e, portanto discriminaram, } \mathrm{y}=\mathrm{x}+2 \text { de } \mathrm{y}=2 \mathrm{x} ; \\ 14 & \text { acertaram os cinco itens. }\end{array}$

Nas duas populações, somente $1 / 4$ dos alunos distinguem $y=x+2$ e $y=2 x$ e menos de um aluno em cada cinco acertaram os cinco itens. Mas, o resultado mais espetacular é que os 165 alunos do premier mesmo tendo passado por esta prova, somente 99 (60\%) deles viam uma diferença de sentido na inclinação da reta associada à diferença entre $y=x \mathrm{e}=-\mathrm{x}$.

Não iremos epilogar estes resultados que mostram de forma muito forte o fosso que separa a abordagem ponto a ponto da abordagem de interpretação global. Esta última exige uma discriminação de todas as varáveis visuais pertinentes, o que não é requisitado e nem induzido pela construção de retas a partir da sua equação. E sem esta abordagem de interpretação global, não existe utilização de gráficos possíveis para dar um valor intuitivo à expressão algébrica que exprima analiticamente as propriedades geométricas ou, a fortiori, para interpretar gráficos cujos eixos representam grandezas heterogêneas (tempo, distância percorrida, velocidade, etc.). 


\subsection{Variáveis visuais gerais e representação de relações}

Hajri (1987) propôs algumas situações elementares nas quais é preciso levar em conta as variáveis gerais. Para um grupo de alunos do troisième e do seconde, levantou as seguintes questões em relação ao plano referencial:

- O conjunto dos pontos que possuem uma abscissa positiva;

- O conjunto de pontos que possuem uma ordenada negativa;

- O conjunto de pontos em que a abscissa e a ordenada possuem o mesmo sinal;

- O conjunto de pontos em que a ordenada é superior à abscissa.

Retomamos este tipo de tarefa fazendo algumas modificações (figura 2 a seguir). Tendo hachurado sobre o plano referencial as zonas correspondentes às expressões abaixo, pedimos aos alunos que, dentre as expressões algébricas $\mathrm{y}=\mathrm{x}, \mathrm{y}>\mathrm{x}, \mathrm{y}<\mathrm{x}, \mathrm{y}=-\mathrm{x}, \mathrm{xy} \geq 0, \mathrm{x}>0 \mathrm{e}$ $\mathrm{y}<0$, indicassem aquelas representadas em cada uma das zonas hachuradas. Propusemos também as mesmas questões de Hajri (1987) introduzindo a variação seguinte: diante do importante fracasso registrado para a expressão "o conjunto dos pontos em que a ordenada é superior à abscissa", em seu lugar apresentamos duas versões, uma com a reta $\mathrm{y}=\mathrm{x}$ traçada $\mathrm{e}$ designada pela sua equação (linha $\mathrm{D}^{\prime}$ da tabela seguinte); a outra sem alguma indicação como no questionário de Hajri (1987) (linha D da tabela seguinte). Não consideramos os pontos na fronteira, quer dizer, a diferença entre $y>x$ e $y \geq x$. Mostraremos a seguir os resultados observados para 105 alunos do premier. 


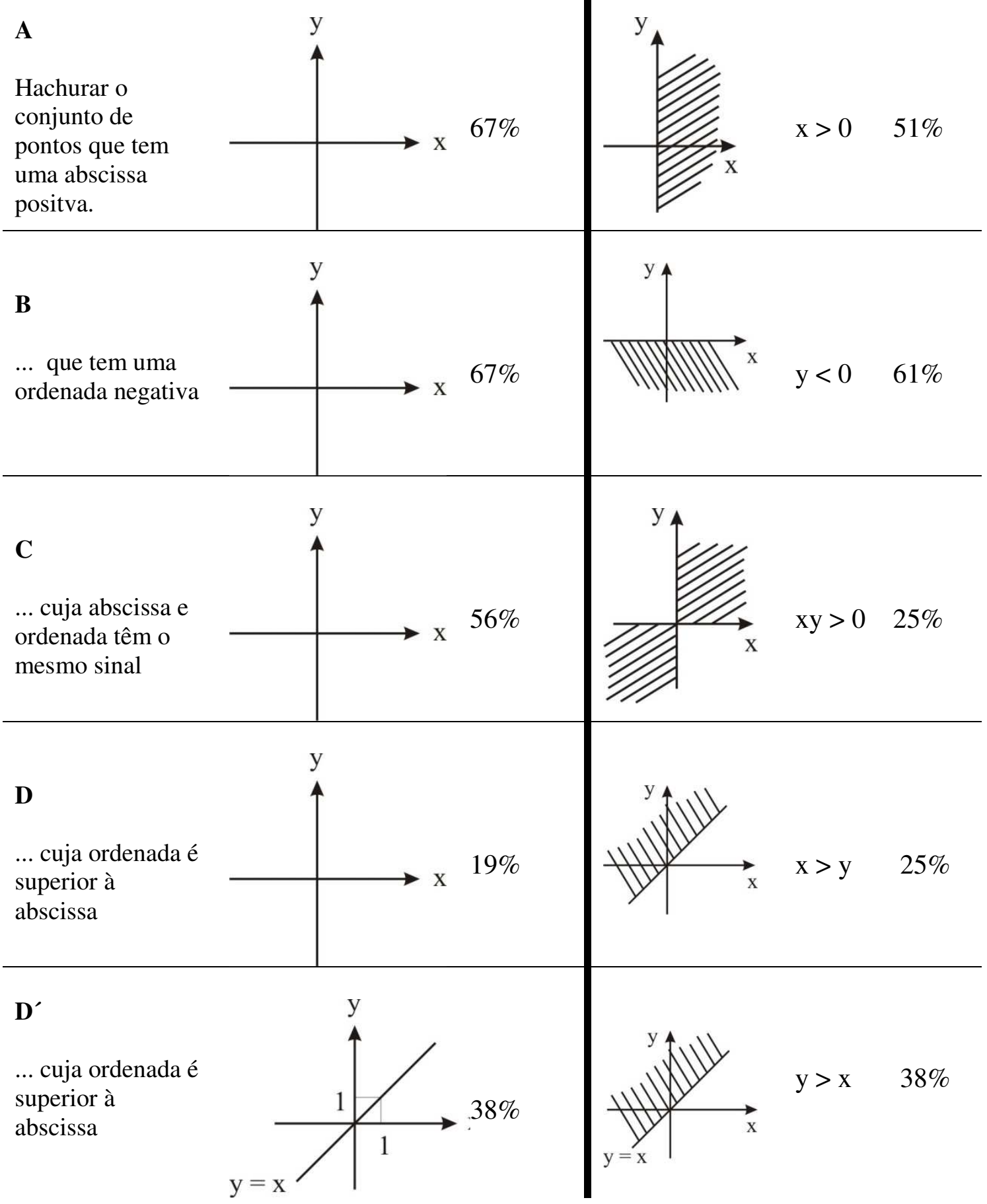

Figura 2: Questões do teste e resultados.

Sobre o primeiro tipo de tarefa (metade à esquerda da tabela), registramos a mesma ordem de acerto e as mesmas diferenças de acerto tal como foram observados por Hajri (1987, p. 95, 96). Se compararmos as diferenças de acerto entre os dois tipos de mudanças de registro solicitados, observamos que:

- Existe congruência semântica para os casos A e B nos dois tipos de tarefas. A expressão discursiva, a representação gráfica e a expressão algébrica dão a mesma 
informação a partir dos mesmos elementos identificadores: abscissa positiva (lado positivo do eixo $\mathrm{x}$ ), ordenada negativa (lado negativo do eixo y). Estas apresentações, que dão conta de três registros diferentes, são congruentes. Os acertos para as quatro questões são da mesma ordem de grandeza;

- Existe ainda congruência semântica para a primeira tarefa do caso $C$, mas não é o caso para a segunda parte. Para reconhecer a equivalência da apresentação algébrica com as duas outras apresentações é preciso recodificar a base de código da expressão: é necessário passar para a equivalência "lados de mesmo sinal" ↔ "o produto das coordenadas é positivo". Quando passamos da primeira para a segunda tarefa, a taxa de acerto cai em mais da metade;

- Não existe mais congruência semântica no caso D: a relação não é mais expressa discursivamente ou algebricamente em função dos elementos identificadores do gráfico (lado positiva do eixo x). A taxa de acerto é deste modo, muito baixa. Por outro lado, a taxa de acerto duplica quando reduzimos a não congruência como no caso da versão $D^{\prime}$.

\subsection{A interpretação de gráficos que representam grandezas heterogêneas.}

Clement assinala o erro do tipo "tomar a altura pela inclinação" na situação da figura 3 a seguir:

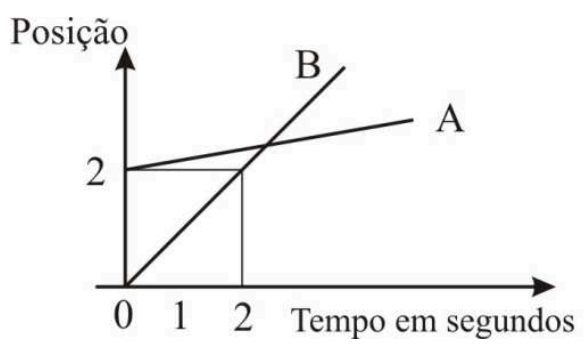

"No instante $\mathrm{t}=2$ segundos, a velocidade do objeto A é maior, menor ou igual à velocidade do objeto B?"

Figura 3: Questão que traz à tona o fenômeno de não congruência semântica por conta da abordagem ponto a ponto.

Fonte: Clement (1985, p. 371, 372).

As observações e as análises precedentes nos esclarecem sobre este tipo de erro geralmente mencionado: 
- A formulação da questão está orientada para a abordagem ponto a ponto. Se a questão tivesse sido formulada de modo a centrar a atenção na consideração dos intervalos no lugar da posição dos dois pontos para $\mathrm{x}=2$, a proporção deste tipo de erro poderia diminuir: durante os dois primeiros segundos, é a velocidade do objeto A que mais aumenta ou a velocidade do objeto B?

- Mas, não é isto a razão essencial deste tipo de erro. Se retomarmos a nossa análise das variáveis visuais, observamos que a altura de um ponto em relação ao eixo vertical não é um valor figural pertinente: a altura resulta de valores tomados por duas variáveis visuais diferentes: o ângulo e a posição do traçado. E sobre o gráfico apresentado, a comparação dos ângulos dos traçados A e B não poderá ser feita se não forem neutralizados os valores diferentes de posição. Isto revela que este tipo de erro não está relacionado à dificuldade do conceito de inclinação, mas à ausência de discriminação das variáveis visuais pertinentes no registro das representações gráficas.

É interessante observar que na experiência de construção de significação das representações gráficas relatadas por Herscovics (1980) que:

- O que está descrito como uma construção intuitiva é simplesmente uma abordagem ponto a ponto (HERSCOVICS, 1980, p. 36).

- A construção racional não leva em conta jamais a articulação entre as variáveis visuais do gráfico e as unidades significativas da expressão simbólica. O registro da representação e o registro da expressão das fórmulas são tratados separadamente e de modo sincrético. (HERSCOVICS, 1980, p. 366-371).

Não é surpreendente que tenha havido fracasso na questão apresentada em 3.3 e, portanto não discriminante $^{7}$ no teste de junho. (HERSCOVICS, 1980, p. 376-377). Esta experiência ignorou os problemas específicos de articulação entre dois registros diferentes de informação.

\footnotetext{
${ }^{7}$ N. do Tradutor. Segundo F. Pluvinage (Analyse des correspondances et questionnaires à modalités. Analyse des Donnés, tome 2. Brochure APMEP, 40. Paris, 1980) as questões podem ser classificadas, do ponto de vista de estrutura do conjunto da população interrogada e do questionário, em quatro tipos: discriminantes (questões em que "alunos fortes" acertam e os "alunos fracos" não acertam), difíceis, fáceis e neutras.
} 


\section{Conclusão}

A interpretação das representações gráficas cartesianas depende de uma identificação precisa de todos os valores das variáveis visuais pertinentes e do reconhecimento qualitativo das unidades da expressão simbólica correspondente. Para uma grande maioria de alunos, esta aprendizagem não se faz apenas por meio do treinamento com exercícios de construção no plano referencial, nem por meio de tarefas de leitura que envolve a associação ponto/coordenadas. A maioria dos alunos limita-se a uma abordagem sincrética e inoperante sem real valor intuitivo das representações gráficas.

Para efetuar uma aprendizagem específica, é importante de distinguir dois tipos de situação quando da utilização das representações gráficas:

- Aquelas em que o recurso aos gráficos se mantém em um quadro puramente matemático: são as relações entre números que são representadas;

- Aquelas em que o recurso se faz em um quadro que não é puramente matemático: são relações entre grandezas de natureza diferentes que são representadas (tempo, distância, velocidade, etc.).

Em vista das análises precedentes, parece que a aprendizagem da abordagem de interpretação global não pode se restringir a um estudo puramente matemático, uma vez que é neste quadro que a articulação entre os valores das variáveis visuais e propriedades conceitualmente relativas a estes valores pode ser mostrada por ela mesma. A significação dos gráficos cartesianos e por consequência a sua leitura, depende da percepção desta articulação. Quando o gráfico representa grandezas heterogêneas, a abordagem de interpretação global se desdobra em uma interpretação das grandezas presentes.

A apresentação de um fenômeno físico, econômico ou biológico dá um interesse talvez maior para os gráficos, mas esta apresentação não facilita a apreensão do funcionamento semiótico de um registro, ela ao contrário a pressupõe.

Desse ponto de vista o questionário sobre gráficos utilizados na enquete de Hart (1981) e retomados em Chelsea diagnostic Mathematics tests (HART, 1984) desperta muitas reservas. Praticamente todas as questões situam-se em um quadro não matemático exigindo a interpretação de grandezas heterogêneas e, as raras questões que se colocam em um quadro puramente matemático, ficam aquém de tarefas que de fato fazem a discriminação entre as variações visuais e as variações correspondentes na expressão algébrica. Para avançarem, as pesquisas sobre as representações gráficas devem tomar outro caminho. 


\section{Bibliografia}

BERTIN, J. Le graphique et le traitement graphique de l'information. Paris: Flammarion, 1977.

CLEMENT, J. Misconceptions in graphing. In: PME 85, p. 369-375, 1985.

HART, K. M et alli. Children's understanding mathematics: 11 - 16 ans. London: John Murray, 1981.

HART, K. M et alli. Chelsea diagnostic Mathematics tests. NFER-NELSON, 1984.

HAJRI, H. Perceptions des relations dans le plan repéré. Thèse de 3ème cycle. Strasbourg: Publication IRMA, 1986.

HERSCOVICS, N. Constructing Meaning for linear equations: a problem of representation. In: RDM, v1.3, p. 351-383, 1980.

Mathématiques $2^{\text {ème }}$. Publ. IREM/Strasbourg. Paris: Casteilla, 1986.

ROGALSKI, J. Caractères réducteurs et producteurs des représentations graphiques des fonctions. Séminaire de Didactique des Mathématiques. Strasbourg, decembre, 1981. 